\title{
Genetic identification of Thunnus orientalis, T. thynnus, and $T$. maccoyii by a cytochrome $b$ gene analysis
}

\author{
Mei-Chen Tseng • Jen-Chieh Shiao • \\ Yin-Huei Hung
}

Received: 18 March 2010 / Accepted: 12 December 2010

(C) Springer Science+Business Media B.V. 2011

\begin{abstract}
The three species of bluefin tunas, Thunnus orientalis, T. maccoyii, and T. thynnus, are morphologically similar, which can pose problems for fisheries management and marketing. We examined intraspecific genetic diversity and interspecific genetic boundaries among these three species by analyzing the cytochrome (Cyt) $b$ gene. The full lengths of the nucleotide sequences were $1,141 \mathrm{bp}$ in $T$. orientalis and T. thynnus and ranged 1,138 1,141 bp in $T$. maccoyii. Mean nucleotide diversities were $0.0019 \pm$ 0.0002 in $T$. thynnus $(n=8), 0.0063 \pm 0.0005$ in $T$. orientalis $(n=22)$, and $0.0059 \pm 0.0007$ in T. maccoyii $(n=24)$. Average numbers of nucleotide differences and nucleotide substitutions per site among the three species were $18.748 \pm 2.879$ and $0.017 \pm 0.003$, respectively. The Neighbor-joining and minimum-evolution trees showed distinct clades with high bootstrapping value support, and the high $F$ st value indicated significant differentiation among the three species. $T$. thynnus, T. orientalis, and T. maccoyii could be
\end{abstract}

Mei-Chen Tseng and Jen-Chieh Shiao contributed equally to this work.

M.-C. Tseng $(\bowtie) \cdot$ Y.-H. Hung

Department of Aquaculture, National Pingtung University of Science and Technology,

Pingtung 912 Taiwan, Republic of China

e-mail: mctseng@mail.npust.edu.tw

J.-C. Shiao

Institute of Oceanography, National Taiwan University,

Taipei 106 Taiwan, Republic of China individually distinguished from each other Thunnus tunas by the 132nd, 375th, and 1,023rd sites of the Cyt $b$ sequences. In the mismatch analysis, Fu's and Tajima's tests of sequences from $T$. orientalis and $T$. maccoyii provided evidence of their population expansion dating to the middle Pleistocene.

Keywords Middle Pleistocene · Nucleotide diversity · Population expansion · Tuna

\section{Introduction}

Thunnus South, 1845 contains eight species which are important commercial and recreational fisheries throughout the tropical and temperate oceans of the world. Thunnus thynnus (Linnaeus, 1758), T. maccoyii (Castelnau, 1872), and T. orientalis (Temminck and Schlegel, 1844) are bluefin tunas which are morphologically similar but have different geographic distributions (Collette et al. 2001). In the West Atlantic, T. thynnus is found from Brazil to Newfoundland (Porch 2005); in the East Atlantic, it is found from roughly the Canary Islands to south of Iceland, and throughout the Mediterranean Sea. Thunnus maccoyii is solely distributed in temperate and cold areas of the Indian Ocean between $30^{\circ} \mathrm{S}$ and $50^{\circ} \mathrm{S}$, and to nearly $60^{\circ} \mathrm{S}$ and in the southwestern Pacific Ocean around Australia and New Zealand (Caton 1991). Thunnus orientalis is primarily found in the North Pacific i.e., the Gulf of Alaska to southern California, Baja 
California, and the area from Sakhalin Island in the southern Sea of Okhotsk to the northern Philippines (Bayliff 2001). It was also caught in the Gulf of Papua, New Guinea (Collette and Smith 1981) and is occasionally caught in Australia, the Galapagos Islands, and New Zealand (Bayliff 1994; Ward et al. 1995; Smith et al. 2001).

Atlantic bluefin tuna has an entirely different distribution from other two species. Gibbs and Collette (1967) described how the anatomical characters of Atlantic and Pacific bluefin tunas are extremely similar and suggested that they should be defined as subspecies. Subsequently, Collette (1999) indicated that the Pacific bluefin is a full species based on genetic and morphological data. Even though the habitats of the two species of Pacific and Southern bluefin tunas partially overlap, they can generally be distinguished by the position of the first ventrally directed parapophysis, i.e., on the 9th vertebra in Southern bluefin but on the 8th vertebra in Pacific bluefin, and the yellow caudal keel in Southern bluefin vs. a dark one in Pacific bluefin. However, distinguishing the Pacific bluefin from the Southern bluefin based on the color of the caudal keel alone is insufficient since this superficial character is not stable (Collette and Nauen 1983, Anonymous 1994, Smith et al. 1994). As mentioned above, these three bluefin tunas are morphologically similar and may be misidentified, posing problems for fisheries management and marketing.

Molecular techniques have long been used to identify fish specimens and explore the population structure of tuna (Ward et al. 1997; Alvarado-Bremer et al. 1998; Chow et al. 2000; Takeyama et al. 2001; Bottero et al. 2007). Protein electrophoresis and restriction fragment length polymorphism (RFLP) of mitochondrial (mt)DNA are the most commonly used methods in published reports. Recently, mtDNA sequences, especially the cytochrome (Cyt) $b$ gene, are frequently utilized to study species identification and historical demography. For example, Tseng et al. (2009) successfully distinguished two morphologically similar Acanthopagrus species using the Cyt $b$ gene, and some similar methodologies were also published by Akimoto et al. (2006). Ward et al. (1995) reported one diagnostic locus (s $A H^{*}$ ) and two nearly diagnostic loci $\left(A D A^{*}\right.$ and $\left.G D A^{*}\right)$ from an allozyme analysis and species-diagnostic restriction digest profiles used to explore genetic differences between Pacific and Southern bluefin tunas. Their conclusions indicated that an mtDNA analysis can provide more-reliable discrimination of specimens than would an allozyme analysis.

In addition, exploring the genetic diversity of species will help elucidate their historical population dynamics (Zhao et al. 2008). The patterns and amounts of diversity in DNA sequences are informative in inferring the history of a population as well as the mechanisms responsible for generating and maintaining polymorphisms ( $\mathrm{Li}$ 1997). In other words, dramatic changes in the environment may leave imprints in the gene pool of a population.

In the Pleistocene, periodic climatic oscillations greatly changed species' geographical distributions and abundances, which likely influenced the genetic diversity of many species (Avise 2000; Hewitt 2000). The development of methods to test mutation-drift equilibrium (MDE) allows the historical demography of a population to be traced using mtDNA data. Mismatch distributions, Fu's $F$ s, and Tajima's $D$ tests were proposed to resolve the population history (Tajima 1989; Fu 1997; Schneider and Excoffier 1999; Wu et al. 2009). The Indian and Pacific Oceans appear to provide great natural settings to study historical dynamics of marine species due to the great abundance of species diversity in these oceans.

The main objective of this study was to use molecular characters of the mtDNA Cyt $b$ gene to discriminate among T. thynnus, T. orientalis, and $T$. maccoyii and estimate interspecific and intraspecific genetic diversity. Moreover, we also explored the historical demography of $T$. orientalis and $T$. maccoyii.

\section{Materials and methods}

\section{Sampling}

Twenty-two $T$. orientalis individuals were caught from the northwestern Pacific Ocean $\left(26^{\circ} \mathrm{N}, 121^{\circ} 45^{\prime} \mathrm{E}\right)$ in 2007 and their tissue samples were collected from the Tongkung fish market, southwestern Taiwan. Twentyfour T. maccoyii individuals were obtained from the Indian Ocean by Taiwanese fisheries observers in 2006 and 2007 as described by Shiao et al. (2008, 2009). Species were primarily identified by the color of the caudal keel and sampling location (Collette and Nauen 1983). Muscle tissues were preserved in $95 \%$ ethanol until the DNA was extracted. 
DNA isolation and polymerase chain reaction (PCR) amplification

Crude DNA was extracted from 500-mg samples of skeletal muscle using the method of Kocher et al. (1989). The complete Cyt $b$ gene was amplified using the forward primer, 5'-ACCAGGACTAATGGCTTG3', and reverse primer, 5'-AGGATTTTAACCTCCG ACGTC-3', which were developed in this study by referring to the complete mtDNA sequence of $T$. thynnus (Manchado et al. 2004). A PCR consisted of approximately $5 \mathrm{ng}$ genomic DNA, 50 pmol each of the forward and reverse primers, $25 \mathrm{mM}$ dNTP, 0.05 $0.1 \mathrm{mM} \mathrm{MgCl} 2,10 \times$ buffer, and $5 \mathrm{U}$ Taq polymerase (Takara Shuzo, Shiga, Japan) brought to $125 \mu$ with Milli-Q water. The PCR program included one cycle of $5 \mathrm{~min}$ at $95^{\circ} \mathrm{C}$; and 38 cycles of $1 \mathrm{~min}$ at $95^{\circ} \mathrm{C}$, $1 \mathrm{~min}$ at $54^{\circ} \mathrm{C}$, and $1.5 \mathrm{~min}$ at $72^{\circ} \mathrm{C}$; followed by a single further extension of $10 \mathrm{~min}$ at $72^{\circ} \mathrm{C}$. We evaluated $10 \mu \mathrm{l}$ of each product on a $0.8 \%$ agarose gel to check the PCR success and confirm the product sizes. The remaining PCR products were run on $0.8 \%$ agarose gels and purified using a DNA Clean/ Extraction kit (GeneMark, Taichung, Taiwan). Purified DNA was subcloned into the pGEM-T easy vector (Promega, Madison, WI, USA) and transformed into the Escherichia coli JM109 strain. Plasmid DNA was isolated using a mini plasmid kit (Geneaid, Taichung, Taiwan). Clones from 22 and 24 individuals of $T$. orientalis and T. maccoyii, respectively, were sequenced on an Applied Biosystems (ABI, Foster City, CA, USA) automated DNA sequencer 377 (vers. 3.3) using a Bigdye sequencing kit (Perkin-Elmer, Wellesley, MA, USA). The T7 and SP6 primers were used in the sequencing reaction, and the PCR cycle parameters for sequencing were 35 cycles of $30 \mathrm{~s}$ at $95^{\circ} \mathrm{C}, 30 \mathrm{~s}$ at $50^{\circ} \mathrm{C}$, and $1 \mathrm{~min}$ at $72^{\circ} \mathrm{C}$.

Cyt $b$ gene analysis

In total, 46 Cyt $b$ sequences subcloned from $T$. orientalis and T. maccoyii were deposited in GenBank (accession nos.: T. orientalis, AM989952 73 and $T$. maccoyii, AM989928 51). Eight homologous sequences of $T$. thynnus derived from NCBI (NC_004901, NC_014052, EF427612.1, EF439243.1, EF439609.1, EU036522.1, AY302574.2, and AB097669.1) were selected as reference sequences in this study. All sequences were aligned using Clustal W (Thompson et al. 1994) and then checked with the naked eye. Different nucleotide ratios within the 54 sequences were estimated. Intraspecific and interspecific genetic diversities of nucleotides were calculated using the MEGA software (Tamura et al. 2007). The general time-reversible plus invariant sites plus gamma distributed model $(\mathrm{GTR}+\mathrm{I}+\mathrm{G})$ is the best-fitting model for DNA substitutions as determined by the Modeltest 3.7 program (Posada and Crandall 1998) using the Akaike information criterion ( $\mathrm{AIC}=5370.45)$. The phylogenetic tree of nucleotide sequences was constructed using Neighbor-joining (NJ) and minimum-evolution (ME) methods with an interior-branch test (Nei and Kumar 2000). The confidence of the clusters was assessed using a bootstrap analysis with 1,000 replications. The minimum spanning tree (MST) was computed from the matrix of pairwise distances between all pairs of haplotypes in each sample using a modification of the algorithm described by Rohlf (1973). Amino acid sequence divergence was estimated using Poisson correction methods (Nei and Kumar 2000) in MEGA (Tamura et al. 2007). The phylogenetic tree of amino acid sequences was constructed by Neighbor-joining method with an anterior-branch test, and the confidence of the clusters was assessed by 1,000 bootstrap replications.

We evaluated whether sequences had evolved under strict neutrality. Fu's Fs (Fu 1997) and Tajima's neutrality tests (Tajima 1989) were performed in Arlequin 3.1 (Excoffier et al. 2005). The significance of the statistics was tested by generating random samples under the hypothesis of selective neutrality and population equilibrium, using a coalescent simulation algorithm adapted from Hudson (1990). Tajima's test is based on an infinite-site model without recombinations. A significant $D$ value can be due to factors other than selective effects, like population expansion, a bottleneck, or heterogeneity of mutation rates (Tajima 1996).

The possible occurrence of historical demographic expansions was examined using the mismatch distribution (Schneider and Excoffier 1999) implemented in Arlequin (Excoffier et al. 2005). The distribution is unimodal in samples following a population demographic expansion (Rogers and Harpending 1992; Rogers 1995). The parameter of demographic expansion, $\tau$, is estimated by a generalized nonlinear leastsquares approach, and confidence intervals of the parameter are computed using a parametric bootstrap 
approach (Schneider and Excoffier 1999). $\tau$ is an index of time since expansion and is expressed in units of mutational time (Slatkin and Hudson 1991). We transformed the value of $\tau$ to an estimate of time since expansion using the equation, $\tau=2 u t$, where $u$ is the mutation rate per locus per generation and $t$ is the number of generations since the expansion. The value of $\mathrm{u}$ is derived from $\mathrm{u}=\mu \mathrm{k}$, where $\mu$ is the mutation rate per nucleotide per generation, and $\mathrm{k}$ is the number of nucleotides in the sequence. The significance of the estimate was obtained by calculating the sum of the squared deviation (SSD) statistic and the raggedness statistic (Harpending 1994). For each statistic, the sudden expansion model was rejected when $p<0.05$ (Excoffier et al. 2005).

\section{Results}

The total length of the Cyt $b$ gene was $1,138 \sim 1,141$ bp among specimens of $T$. maccoyii and 1,141 bp among specimens of $T$. orientalis and $T$. thynnus. The percentages of the nucleotide composition did not significantly differ among the three bluefin tuna species. Altogether seven polymorphic sites occurred within eight sequences of T. thynnus. Values of $H_{d}, \mathrm{k}$, and $\mathrm{Pi}$ within these sequences were 1, 2.214, and $0.0019 \pm 0.0002$. In total, 51 polymorphic sites and 11 parsimoniously informative sites occurred within the 22 sequences of $T$. orientalis (Fig. 1). Haplotype diversity $\left(H_{d}\right)$, the average number of nucleotide differences $(\mathrm{k})$, and the nucleotide diversity $(\mathrm{Pi})$ within these sequences were 1, 7.528, and $0.0066 \pm 0.0004$, respectively. Forty-eight polymorphic sites and eight parsimoniously informative sites occurred within the 24 sequences of $T$. maccoyii. All sequences of $T$. maccoyii in this study were diverse except for one identical haplotype found in three specimens ( $\mathrm{S} 01$, $\mathrm{S} 03$, and S05). Values of $H_{d}, \mathrm{k}$, and Pi within the 24 sequences of $T$. maccoyii were $0.917,6.706$, and $0.0059 \pm 0.0007$, respectively (Table 1 ). There were 28 fixed differences within specimens of T. maccoyii. Six mutations were common between $T$. orientalis and $T$. maccoyii (Fig. 1). The number of nucleotide substitutions ranged $2 \sim 13$ among haplotypes of $T$. orientalis, $1 \sim 15$ among haplotypes of $T$. maccoyii, 1 3 among haplotypes of $T$. thynnus, and $8 \sim 47$ among the three species. The $\mathrm{k}$ value and average number of nucleotide substitutions per site $\left(D_{\mathrm{xy}}\right)$ between $T$. orientalis and $T$. maccoyii were 40 and $0.036 \pm 0.0023$, respectively. Base pair 831 had different compositions in the three bluefin tuna: it was thymidine in Pacific bluefin, guanine in southern bluefin, and adenine in Atlantic bluefin. The base pair 32 guanine was solely present in T. thynnus but not in the other two Thunnus species.

Intraspecific Kimura-2-parameter genetic distances among different haplotypes ranged $0.001 \sim 0.020$ for $T$. maccoyii, $0.002 \sim 0.012$ for T. orientalis, and $0.001 \sim$ 0.003 in $T$. thynnus. The interspecific distances ranged from 0.007 (T. maccoyii vs. T. thynnus) to 0.043 ( $T$. maccoyii vs. T. orientalis) among the three bluefin tuna species (Table 2). The mean nucleotide diversity among the three species ranged from $0.009 \pm 0.002$ ( $T$. maccoyii vs. T. thynnus) to $0.037 \pm 0.005$ (T. maccoyii vs. $T$. orientalis). The coefficients of differentiation among the three species were $0.706 \pm 0.04$ (T. orientalis vs. T. maccoyii), $0.742 \pm 0.04$ ( $T$. orientalis vs. $T$. thynnus), and $0.436 \pm 0.07$ (T. maccoyii vs. T. thynnus). Specimens of T. orientalis, T. thynnus, and T. maccoyii were distributed in three monophyletic clades in the $\mathrm{NJ}$ and ME phylogenetic tree, with $99 \%, 98 \%$, and $81 \%$ bootstrap support, respectively (Fig. 2). Atlantic bluefin tuna and southern bluefin tuna were shown to be sister taxa.

The Cyt $b$ gene is highly conserved and codes a protein of 380 amino acids. After alignment, four identical amino acid sequences were observed in four specimens of $T$. orientalis and 11 specimens of $T$. maccoyii. Eight sequences of T. thynnus from NCBI's GenBank coded an identical amino acid sequence. In total, 39 polymorphic amino acid sites were found within 36 different sequences from the three bluefin tuna species (Fig. 3). The intraspecific Poisson correction distances of different amino acid sequences within T. orientalis as well as in T. maccoyii ranged $0.003 \sim 0.016$. Interspecific genetic distances among the three bluefin tuna species ranged 0.003 ( $T$. maccoyii vs. T. thynnus) $\sim 0.021$ (T. maccoyii vs. T. orientalis) (Table 2). Mean distances within specimens of $T$. orientalis and T. maccoyii were $0.008 \pm$ 0.002 and $0.007 \pm 0.002$, respectively. Interspecific mean distances ranged from $0.007 \pm 0.003$ (T. maccoyii vs. T. thynnus) to $0.013 \pm 0.004$ (T. orientalis vs. T. maccoyii). Most of the nucleotide substitutions observed between sequences were silent mutations. However, the 125th and 341 st sites of the amino acid sequence had specific compositions among $T$. orientalis (Met and Ile), T. maccoyii (Ile and Met), and T. 
Fig. 1 Variable sites at aligned nucleotide sequences of the cytochrome $b$ gene for 46 specimens of Thunnus orientalis $(\mathrm{N})$ and $T$. maccoyii (S). The eight sequences of $T$. thynnus were obtained from NCBI's GenBank

thymus (Met and Met). In total, 22 and 17 private amino acid variations were present in $T$. orientalis and T. maccoyii, respectively. Only one common variation occurred at the 380th position (Fig. 3). The NJ topology of amino acid sequences indicated a failure to resolve interrelationships among these species (Fig. 4). Thunnus thynnus has a closer relationship to T. maccoyii than to T. orientalis based on the data of nucleotide sequences, but the reverse situation occurred in the analytic results from the amino acid sequences.

Mismatch analyses of the Cyt $b$ data indicated a recent population expansion in both species. Significant $(p<0.02$ and $p=0)$ negative values of Tajima's $D$ $(-1.824$ and -1.982$)$ and Fu's $F_{\mathrm{s}}(-17.664$ and -15.286$)$ were present in $T$. orientalis and $T$. maccoyii, respectively. In particular, the $F_{\mathrm{s}}$ value is very sensitive to recent population demographic expansions, which generally lead to large negative values.
A plot of the mismatch distribution showed that the mean numbers of pairwise differences were 3.761 for T. orientalis and 3.912 for T. maccoyii, and showed that they were unimodal (Fig. 5), indicating an expanding population. The sudden expansion model parameters in $T$. orientalis were $\tau=7.496(97.5 \%$ confidence limits (CLs): 4.941 8.965) and in $T$. maccoyii were $\tau=5.273 \quad(97.5 \%$ CLs: $1.664 \sim$ 14.316). The SSDs between the observed and expected mismatch and raggedness index were $0.0042(p=0.52)$ and $0.0175(p=0.35)$ in T. orientalis; and were $0.0055(p=0.60)$ and $0.0092(p=0.92)$, in $T$. maccoyii, respectively. All data indicated that the sudden expansion model could not be rejected. The MSTs of T. orientalis and T. maccoyii were characterized by a reticulate phylogeny (Fig. 6). Most of haplotypes were located at the tips.

The generation time estimated for $T$. orientalis was roughly 5 years and for $T$. maccoyii was roughly $10 \sim$ 


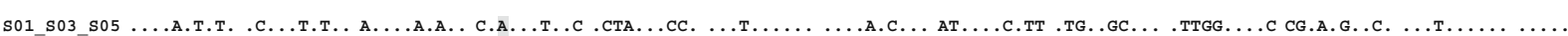

S06

S07

S08

S09

S10

S11

S12

$\mathrm{S} 13$

S14

S15

S16

S17

S18

S19

S20

S21

S22

S23

S24

AB097669.1

АY3 02574.2

EF427612.1

EF439243.1

EF439609.1

EU036522.1

NC_004901

NC_014052

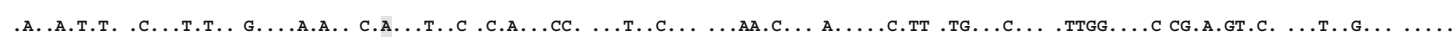

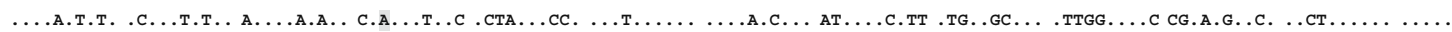

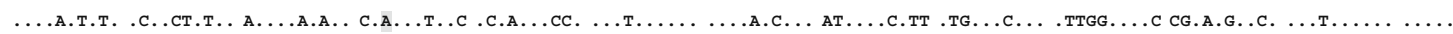

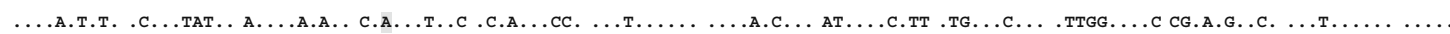

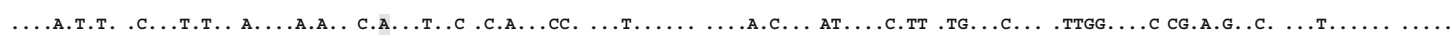

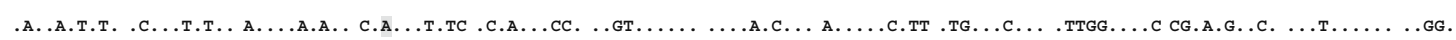

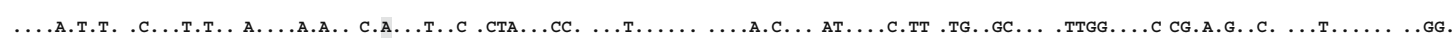

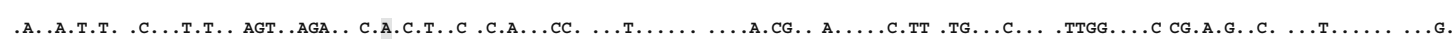

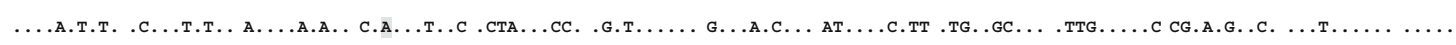

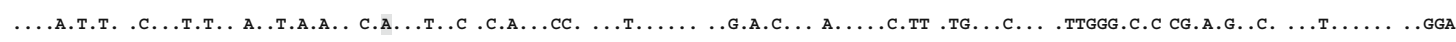

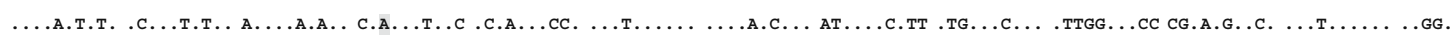

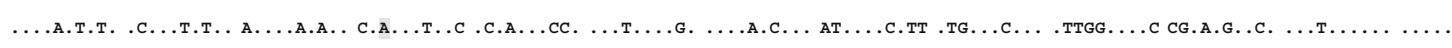

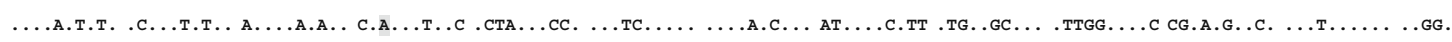

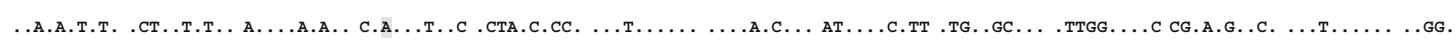

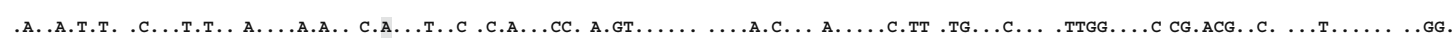

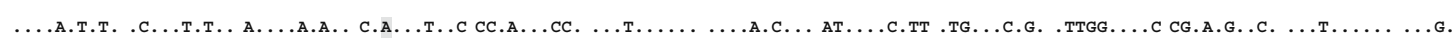

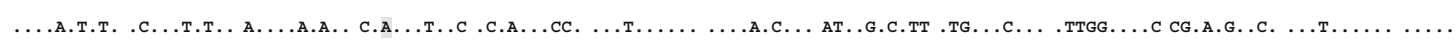

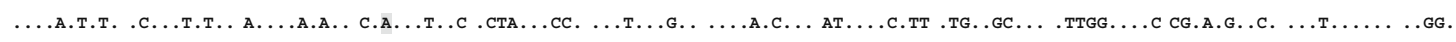

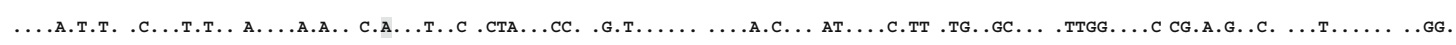

Fig. 1 (continued)

12 years (Cascorbi 2003; Gunn et al. 2008), which is concordant with ecological data (Hattour and Macías 2002; Mather et al. 1995). The estimated time since the population expansion began, calculated from $t=\tau$ / $2 \mathrm{u}$, was roughly 0.1328 Mya for $T$. orientalis and $0.1868 \sim 0.2242$ Mya for T. maccoyii.

\section{Discussion}

Thunnus thynnus and T. orientalis should be classified into two separate species. Gibbs and Collette (1967) indicated that $T$. orientalis and T. thynnus should be considered subspecies based on the comparative

Table 1 Intraspecific and interspecific nucleotide differences $(\mathrm{k})$ (above the diagonal) and nucleotide diversity (Pi) (below the diagonal) based on mitochondrial cytochrome $b$ sequence data

\begin{tabular}{|c|c|c|c|c|c|c|c|c|c|c|c|c|c|c|c|c|c|c|c|c|c|c|}
\hline & 1 & 2 & 3 & 4 & 5 & 6 & 7 & 8 & 9 & 10 & 11 & 12 & 13 & 14 & 15 & 16 & 17 & 18 & 19 & 20 & 21 & 22 \\
\hline 1. N02 & **** & 2 & 5 & 5 & 11 & 5 & 8 & 42 & 37 & 37 & 40 & 39 & 41 & 43 & 37 & 37 & 38 & 37 & 36 & 37 & 37 & 37 \\
\hline 2. N03 & 0.0018 & $* * *$ & 5 & 5 & 11 & 5 & 8 & 44 & 39 & 39 & 42 & 41 & 43 & 45 & 39 & 40 & 40 & 39 & 38 & 39 & 40 & 39 \\
\hline 3. N04 & 0.0044 & 0.0044 & $* * *$ & 2 & 8 & 4 & 7 & 41 & 36 & 36 & 41 & 38 & 40 & 42 & 36 & 37 & 37 & 36 & 35 & 36 & 37 & 36 \\
\hline 4. N08 & 0.0044 & 0.0044 & 0.0018 & **** & 8 & 4 & 7 & 41 & 36 & 36 & 41 & 38 & 40 & 42 & 36 & 37 & 37 & 36 & 35 & 36 & 37 & 36 \\
\hline 5. N14 & 0.0096 & 0.0096 & 0.0070 & 0.0070 & $* * *$ & 6 & 9 & 43 & 38 & 38 & 43 & 40 & 42 & 44 & 42 & 43 & 43 & 42 & 41 & 42 & 43 & 41 \\
\hline 6. N17 & 0.0044 & 0.0044 & 0.0035 & 0.0035 & 0.0088 & ***** & 9 & 47 & 42 & 42 & 43 & 40 & 42 & 44 & 38 & 39 & 39 & 38 & 37 & 38 & 39 & 38 \\
\hline 7. N22 & 0.0070 & 0.0070 & 0.0061 & 0.0061 & 0.0114 & 0.0061 & $* * *$ & 46 & 41 & 41 & 46 & 43 & 45 & 47 & 41 & 42 & 42 & 41 & 40 & 41 & 42 & 41 \\
\hline 8. S02 & 0.0368 & 0.0386 & 0.0359 & 0.0359 & 0.0412 & 0.0377 & 0.0403 & $* * *$ & 7 & $\begin{array}{l}41 \\
7\end{array}$ & 14 & 9 & 11 & 13 & $\begin{array}{l}\text { 1) } \\
13\end{array}$ & 14 & 14 & $\begin{array}{l}11 \\
13\end{array}$ & 12 & $\begin{array}{l}11 \\
13\end{array}$ & 14 & $\begin{array}{l}41 \\
13\end{array}$ \\
\hline 9. S08 & 0.0324 & 0.0342 & 0.0316 & 0.0316 & 0.0368 & 0.0333 & 0.0359 & 0.0061 & ******* & 2 & 9 & 4 & $\begin{array}{l}6 \\
6\end{array}$ & 8 & 9 & 10 & $\begin{array}{l}14 \\
10\end{array}$ & 9 & 8 & 9 & 10 & 9 \\
\hline 10. S09 & 0.0324 & 0.0342 & 0.0316 & 0.0316 & 0.0368 & 0.0333 & 0.0359 & 0.0061 & 0.0018 & 承** & 9 & 4 & 6 & 8 & 9 & 10 & 10 & 9 & 8 & 9 & 10 & 9 \\
\hline 11. S15 & 0351 & 0368 & 0.0359 & 0.0359 & 0.0377 & 0.0368 & 0.0403 & 0.0123 & 0.0079 & 0.0079 & ***** & 7 & 9 & i1 & 16 & 17 & 17 & 16 & $\begin{array}{l}0 \\
15\end{array}$ & 16 & & 16 \\
\hline 12. S16 & 0324 & 0.0359 & 0.0333 & 0.0333 & 0.0351 & 0.0351 & 0.0377 & 0.0079 & 0.0035 & 0.0035 & 0.0061 & $* * *$ & 4 & 6 & 11 & 12 & 12 & 11 & 10 & 11 & & 11 \\
\hline 13. S18 & 3359 & 0.0377 & 0.0351 & 0.0351 & 0.0368 & 0.0368 & 0.0394 & 0.0096 & 0.0053 & 0.0053 & 0.0079 & 0.0035 & $* * *$ & 4 & 9 & 10 & 10 & & & & & 9 \\
\hline $\begin{array}{l}14.519 \\
14.5\end{array}$ & 0.0377 & 0.0394 & 0.0368 & 0.0368 & $\begin{array}{l}0.0368 \\
0.0368\end{array}$ & 0.0368 & $\begin{array}{l}0.0412 \\
0.0412\end{array}$ & 0.0114 & 0.0070 & 0.0070 & 0.0096 & 0.0053 & 0.0035 & $\stackrel{4 * *}{* * 2}$ & 11 & 12 & $\begin{array}{l}10 \\
12\end{array}$ & 11 & 10 & 11 & 12 & 11 \\
\hline 15. AB097669.1 & 0.0324 & 0.0342 & 0.0316 & 0.0316 & 0.0368 & 0.0333 & 0.0359 & 0.0114 & 0.0079 & 0.0079 & 0.0140 & 0.0096 & 0.0079 & 0.0096 & $* * *$ & 3 & 3 & 2 & 1 & 2 & 3 & 2 \\
\hline 16. AY302574.2 & 0.03 & 0.03 & 0.0324 & 0.0324 & 0.0377 & & 0.0368 & 0.0123 & 0.0088 & 0.0088 & 0.0149 & 0.0105 & 0.0088 & 0.0105 & 0.0027 & $* * *$ & 2 & 3 & 2 & 3 & 0 & 3 \\
\hline 17. EF4276 & 0.0333 & 0.0351 & 0.0324 & 0.03 & 0.0377 & & 0.0368 & 0.0123 & 0.0088 & 0.0088 & 0.0149 & 0.0105 & 0.0088 & 0.0105 & 0.0027 & 0.0018 & **** & 3 & 2 & 3 & 2 & 3 \\
\hline 18. EF4 & 0.03 & 0.03 & 0.03 & 0.03 & 0.03 & & 0.0359 & 0.0114 & 0.0079 & 0.0079 & 0.0140 & 0.0096 & 0.0079 & 0.0096 & 0.0018 & 0.0027 & 0.0027 & $* * *$ & 1 & 2 & 3 & 2 \\
\hline 19. EF439 & 0.03 & 0.0333 & 0.0307 & 0.0307 & 0.0359 & 0.03 & 0.0351 & 0.0105 & 0.0070 & 0.0070 & 0.0131 & 0.0088 & 0.0070 & 0.0088 & 0.0009 & 0.0018 & 0.0018 & 0.0009 & $* * *$ & 1 & 2 & 1 \\
\hline 20. EU 036522.1 & 0.0324 & 0.0342 & 0.0316 & 0.0316 & 0.0368 & 0.0333 & 0.0359 & 0.0114 & 0.0079 & 0.0079 & 0.0140 & 0.0070 & 0.0079 & 0.0096 & 0.0018 & 0.0027 & 0.0027 & 0.0018 & 0.0009 & **** & 3 & 2 \\
\hline 21. NC 004901 & 0.0324 & 0.0351 & 0.0324 & 0.0324 & 0.0377 & 0.0342 & 0.0368 & 0.0123 & 0.0088 & 0.0088 & 0.0149 & 0.0105 & 0.0088 & 0.0105 & 0.0027 & 0.0008 & 0.0027 & 0.0027 & 0.0018 & 0.0027 & $* * * *$ & 3 \\
\hline 22. NC 014052 & 0.0324 & 0.0342 & 0.0316 & 0.0316 & 0.0359 & 0.0333 & 0.0359 & 0.0114 & 0.0079 & 0.0079 & 0.0140 & 0.0096 & 0.0079 & 0.0096 & 0.0018 & 0.0027 & 0.0018 & 0.0018 & 0.0009 & 0.0018 & 0.0027 & *** \\
\hline
\end{tabular}

$\mathrm{N}$ and $\mathrm{S}$ respectively indicate Thunnus orientalis and T. maccoyii.

The data within species are indicated by gray shading 
Table 2 Intraspecific and interspecific nucleotide genetic distances (above the diagonal) and amino acid genetic distances (below the diagonal) based on mitochondrial cytochrome $b$ sequence data

\begin{tabular}{|c|c|c|c|c|c|c|c|c|c|c|c|c|c|c|c|c|c|c|c|c|c|c|}
\hline & 1 & 2 & 3 & 4 & 5 & 6 & 7 & 8 & 9 & 10 & 11 & 12 & 13 & 14 & 15 & 16 & 17 & 18 & 19 & 20 & 21 & 22 \\
\hline $1 . \mathrm{N} 02$ & **** & 0.002 & 0.004 & 0.004 & 0.010 & 0.004 & 0.007 & 0.038 & 0.033 & 0.033 & 0.036 & 0.035 & 0.037 & 0.039 & 0.033 & 0.033 & 0.034 & 0.033 & 0.032 & 0.033 & 0.033 & 0.033 \\
\hline 2. N03 & 0 & **** & 0.004 & 0.004 & 0.010 & 0.004 & 0.007 & 0.040 & 0.035 & 0.035 & 0.038 & 0.037 & 0.039 & 0.041 & 0.035 & 0.036 & 0.036 & 0.035 & 0.034 & 0.035 & 0.036 & 0.035 \\
\hline 3. N04 & 0.005 & 0.005 & $* * *$ & 0.002 & 0.007 & 0.004 & 0.006 & 0.037 & 0.033 & 0.033 & 0.037 & 0.034 & 0.036 & 0.038 & 0.032 & 0.033 & 0.033 & 0.032 & 0.032 & 0.032 & 0.033 & 0.032 \\
\hline 4. N08 & 0.003 & 0.003 & 0.003 & $* * *$ & 0.007 & 0.004 & 0.006 & 0.037 & 0.033 & 0.033 & 0.037 & 0.034 & 0.036 & 0.038 & 0.032 & 0.033 & 0.033 & 0.032 & 0.032 & 0.032 & 0.033 & 0.032 \\
\hline 5. N14 & 0.008 & 0.008 & 0.008 & 0.005 & $* * * *$ & 0.009 & 0.012 & 0.043 & 0.038 & 0.038 & 0.039 & 0.036 & 0.038 & 0.040 & 0.038 & 0.039 & 0.039 & 0.038 & 0.037 & 0.038 & 0.039 & 0.037 \\
\hline 6. N17 & 0.005 & 0.005 & 0.005 & 0.003 & 0.008 & $* * *$ & 0.006 & 0.039 & 0.034 & 0.034 & 0.038 & 0.036 & 0.038 & 0.040 & 0.034 & 0.035 & 0.035 & 0.034 & 0.033 & 0.034 & 0.035 & 0.034 \\
\hline 7. N22 & 0.011 & 0.011 & 0.011 & 0.008 & 0.013 & 0.011 & $* * *$ & 0.042 & 0.037 & 0.037 & 0.042 & 0.039 & 0.041 & 0.043 & 0.037 & 0.038 & 0.038 & 0.037 & 0.036 & 0.037 & 0.038 & 0.037 \\
\hline 8. S02 & 0.016 & 0.016 & 0.016 & & 0.019 & 0.016 & 0.021 & 0.042 & 0.006 & 0.006 & 0.012 & 0.008 & $\begin{array}{l}0.010 \\
0.010\end{array}$ & 0.012 & 0.0 & 0.0 & 0.0 & 12 & 11 & 12 & 0.012 & 0.012 \\
\hline 9. 508 & & 0 & & & 0 & 0.008 & 0.013 & 0.008 & $* * * *$ & 0.002 & 0.008 & 0.004 & 0.005 & 0.007 & 0.0 & 0.00 & 0.009 & 0.0 & 07 & 08 & 0.009 & 0.008 \\
\hline 10. S09 & & & 0.011 & & 0.013 & 0.011 & 0.016 & 0.011 & 0.003 & $* * *$ & 0.008 & 0.004 & 0.005 & 0.007 & 0.0 & 0.009 & 0.009 & 0.0 & 0.007 & 0.008 & 0.009 & 0.008 \\
\hline 11. S15 & 111 & 0.011 & & 008 & 0.011 & 0.011 & 0.016 & 0.011 & 0.003 & 0.005 & $* * * *$ & 0.006 & 0.0 & 0.0 & 0.0 & 0.0 & 0.0 & 0.0 & 0.0 & 4 & 0.015 & 0.014 \\
\hline 12. S16 & & 0.011 & & & 0.011 & & 0.016 & & 0.003 & 0.005 & 0 & $* * *$ & 0.004 & 0.0 & 0.0 & 0.0 & 0.0 & 0.0 & 0.0 & 0.0 & 0.011 & 0.010 \\
\hline 13. S18 & & 0.011 & & & 0.011 & & 0.016 & & & 0.005 & 0 & 0 & $* * *$ & 0.0 & 0.0 & 0.0 & 0.0 & 0.0 & 0.0 & 8 & 99 & 0.008 \\
\hline 14. S19 & 0.013 & 0.013 & 0.013 & 0.011 & 0.013 & 0.013 & 0.019 & 0.0 & 0.06 & 0.008 & 0.003 & 0.003 & 0.003 & $* * *$ & 0.010 & 0.011 & 0.0 & 0.0 & 0.009 & 0.0 & 0.011 & 0.010 \\
\hline 15. АВ097669 & & 0.005 & 0.005 & & 0.008 & 0.00 & 0.0 & $0 . c$ & 0.0 & 0.0 & 0.0 & 0.0 & 0.0 & 0.008 & $* * *$ & 0.003 & 0.0 & 0.0 & 0.0 & 0.0 & 0.003 & 0.002 \\
\hline 16. AY3 & & & & & 0.0 & 0.0 & 0.0 & 0.0 & 0.0 & 0.0 & 0.0 & 0.0 & 0.005 & 0.008 & 0 & $* * *$ & 0.002 & 0.003 & 0.0 & 0.0 & 0 & 0.003 \\
\hline 17. EF427 & & 0.005 & & & 0.00 & 0.00 & 0.011 & 0.0 & 0.00 & 0.0 & 0.0 & 0.005 & 0.005 & 0.008 & 0 & 0 & $* * *$ & 0.003 & 0.002 & 0.003 & 0.002 & 0.003 \\
\hline 18. EF43 & 0.005 & 0.005 & 0.005 & 0.003 & 0.008 & 0.005 & 0.011 & 0.011 & 0.003 & 0.005 & 0.005 & 0.005 & 0.005 & 0.008 & 0 & 0 & 0 & $* * *$ & 0.001 & 0.002 & 0.003 & 0.002 \\
\hline 19. EF4 & 0.005 & 0.005 & 0.005 & 0.003 & 0.008 & 0.005 & 0.011 & 0.011 & 0.003 & 0.005 & 0.005 & 0.005 & 0.005 & 0.008 & 0 & 0 & 0 & 0 & **** & 0.001 & 0.002 & 0.001 \\
\hline 20. EU036522.1 & 0.005 & 0.005 & 0.005 & 0.003 & 0.008 & 0.005 & 0.011 & 0.011 & 0.003 & 0.005 & 0.005 & 0.005 & 0.005 & 0.008 & 0 & 0 & 0 & 0 & 0 & $* * *$ & 0.003 & 0.002 \\
\hline 21. NC & 0.005 & 0.005 & 0.005 & 0.003 & 0.008 & 0.005 & 0.011 & 0.011 & 0.003 & 0.005 & 0.005 & 0.005 & 0.005 & 0.008 & 0 & 0 & 0 & 0 & 0 & 0 & **** & 0.003 \\
\hline 22. NC 014052 & 0.005 & 0.005 & 0.005 & 0.003 & 0.008 & 0.005 & 0.011 & 0.011 & 0.003 & 0.005 & 0.005 & 0.005 & 0.005 & 0.008 & 0 & 0 & 0 & 0 & 0 & 0 & 0 & $* * *$ \\
\hline
\end{tabular}

$\mathrm{N}$ and $\mathrm{S}$ respectively indicate Thunnus orientalis and T. maccoyii. Intraspecific data are indicated by gray shading

anatomy. However, Collette (1999) proposed that Atlantic and Pacific bluefin tunas be regarded as full species based on genetic and morphological evidence. In this study, we found that $T$. thynnus could be distinguished from other Thunnus species based on the 132nd nucleotide of the Cyt $b$ gene $(\mathrm{G})$ which was diagnostic for $T$. thynnus (Fig. 1). The 831st nucleotide of the Cyt $b$ gene differed between T. thynnus and (a)

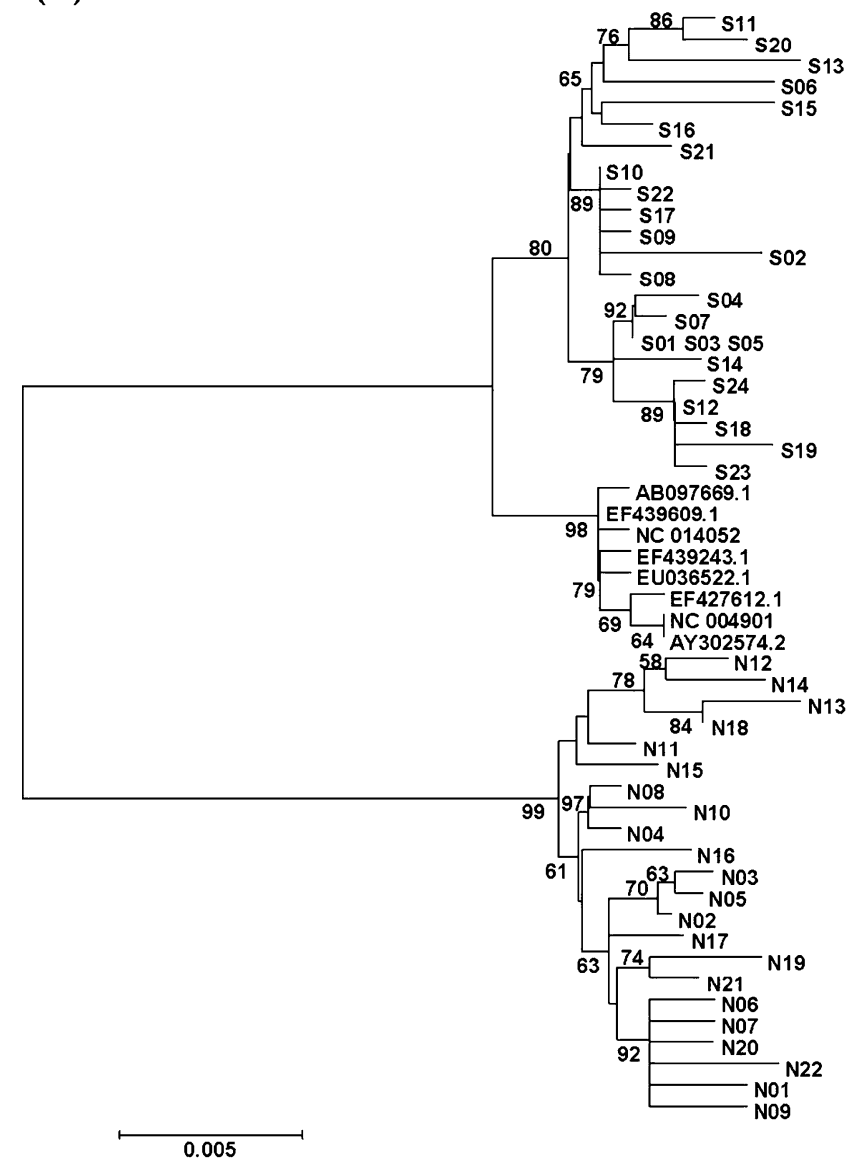

(b)

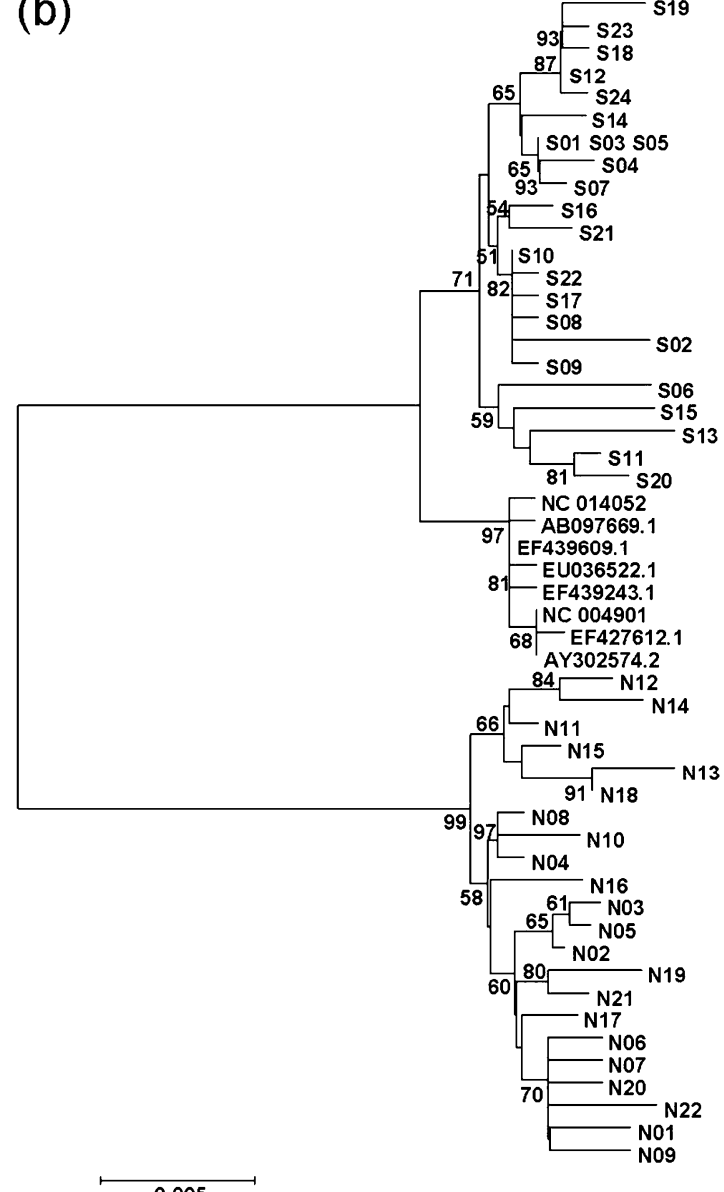

Fig. 2 a Neighbor-joining and $\mathbf{b}$ maximum-evolution phylogenetic trees constructed using the genetic distance method. Bootstrap values of $>50 \%$ (out of 1,000 replicates) are shown at the nodes 
Fig. 3 Polymorphic sites in the 19 amino acid sequences of cytochrome $b$ from Thunnus orientalis $(\mathrm{N})$ and in 16 sequences from $T$. maccoyii (S). Samples N03 and N05 shared an identical sequence with N02. N12 and N18 shared an identical sequence. S01, S03, S05, S08, S10, and S14 had the same sequence. S12, S15, S16, S18, and S24 shared a common sequence. Eight nucleotide sequences from T. thynnus coded an identical amino acid sequence
N11

N13

N14

N15

N16

N17

N12, N18

N19

N20

N21

N22

$\mathrm{S} 02$

S04

s06

S07

s09

s11

513

$\mathrm{S} 01, \mathrm{~s} 03, \mathrm{~s} 05, \mathrm{~s} 08, \mathrm{~s} 10, \mathrm{~s} 14$

$\mathrm{S} 17$

S19

S20

$\mathrm{S} 21$

$\mathrm{S} 22$

S23

$\mathrm{s} 12, \mathrm{~s} 15, \mathrm{~s} 16, \mathrm{~s} 18, \mathrm{~s} 24$

NC_004901 $\left.\begin{array}{llll}11111 & 1111112222 & 2222222233 & 333333333\end{array}\right]$

$\left.\begin{array}{llllll}117801223 & 4467780011 & 3455569924 & 444666778\end{array}\right]$

$\left.\begin{array}{lllll}2696627458 & 4791802724 & 9467825971 & 348458480\end{array}\right]$

AGVGGLVMMM TTSDFAENNN LLFTPMLLTI AEIVSLNGS

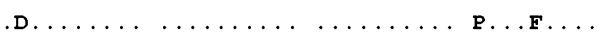

$. \mathbf{y} \ldots \ldots, \ldots \ldots \ldots, \ldots \ldots \ldots, \ldots, \ldots$

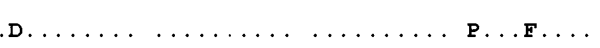

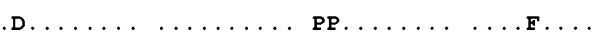

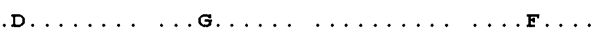

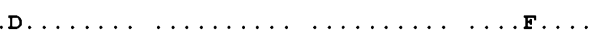

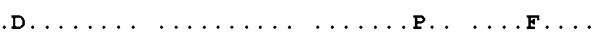

.$D I \ldots \ldots I \ldots \ldots \ldots \ldots \ldots \ldots \ldots$

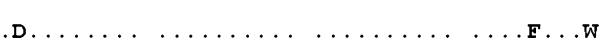

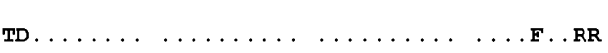

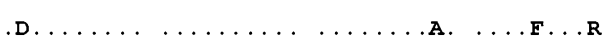

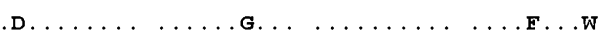

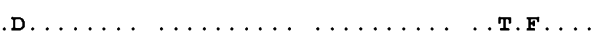

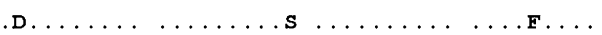

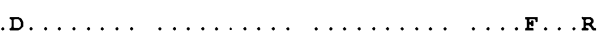

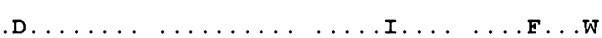

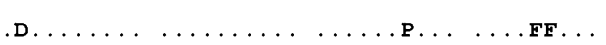

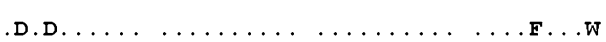

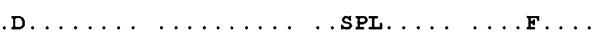

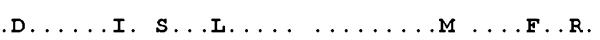

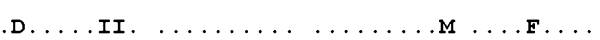

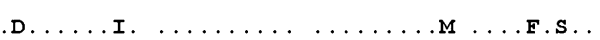

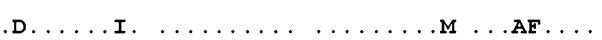

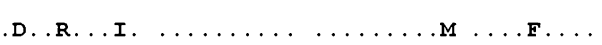

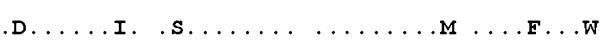

.D. . VG.II $\ldots \ldots \ldots \ldots \ldots$. . . . F . .

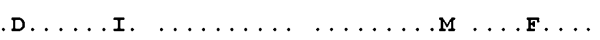

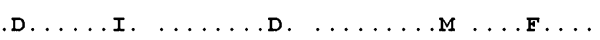

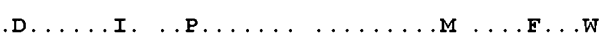

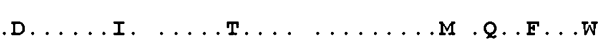

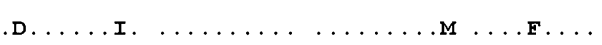

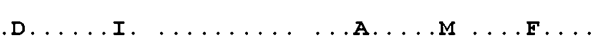

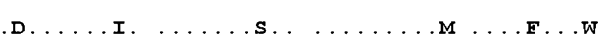

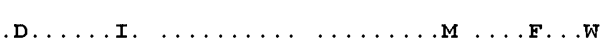

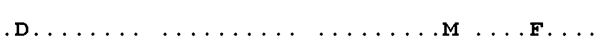

T. orientalis (A vs. T). The $\mathrm{NJ}$ and ME trees also suggested that $T$. thynnus and $T$. maccoyii have a closer phylogenetic relationship than either with $T$. orientalis. The Cyt $b$ gene is also an efficient genetic marker for distinguishing T. orientalis and T. maccoyii from other Thunnus species. For example, the 375th nucleotide (A) is diagnostic for T. maccoyii. The 1023rd nucleotide (A) is diagnostic for T. orientalis
(Fig. 1). Both characters can be used to distinguish the respective species from other Thunnus species. Although these two species have overlapped distributions in the southern Pacific Ocean, results by Chow and Kishino (1995) based on mtDNA ATPase data revealed significant differentiation between the two species. We merely employed eight different Cyt $b$ sequences of $T$. thynnus from NCBI's GenBank, but 
Fig. 4 The Neighborjoining tree of cytochrome

Numbers above the branches indicate the bootstrap values $b$ amino acid sequences.

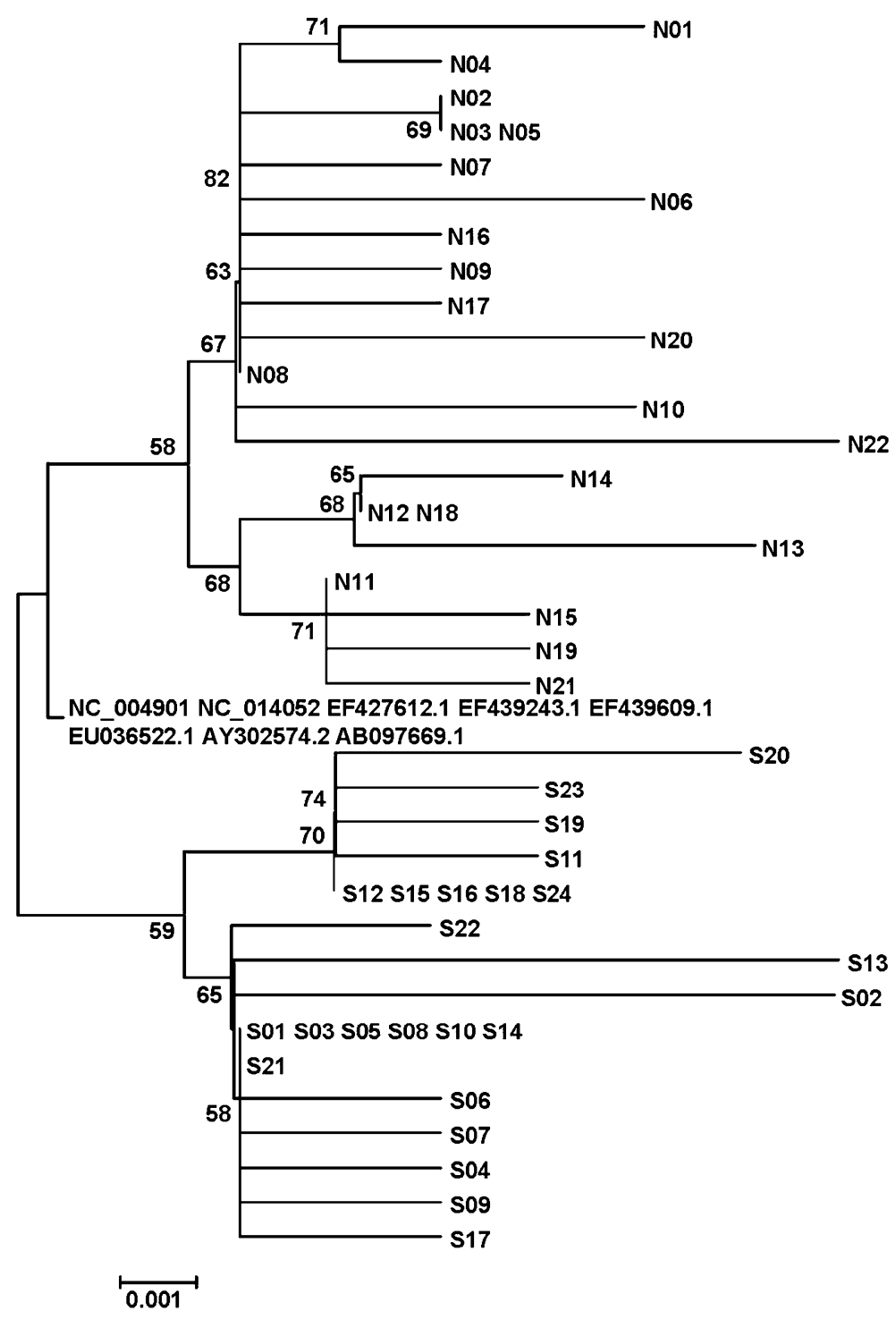

the results clearly showed that the Atlantic, Pacific, and Southern bluefin tunas should be defined as separate species.

Lengths of the complete Cyt $b$ gene in tuna from this study ranged $1,138 \sim 1,141 \mathrm{bp}$ and were similar to those of most fishes (Takehana et al. 2004; Zhao et al. 2008). Compared to the intraspecific nucleotide diversity of other fishes, that of a freshwater salangid was 0.0022 (Zhao et al. 2008), a tropical damselfish Acanthochromis polyacanthus was 0.045 (Planes et al. 2001), a coastal marine bonefish Albula sp. was 0.0036 (Pfeiler et al. 2008), and migratory T. thynnus, T. orientalis, and T. maccoyii were $0.0019,0.0063$, and 0.0059 , respectively (this study). The migratory $T$. orientalis and T. maccoyii have higher nucleotide diversities than do freshwater and coastal fishes. They have a high $H_{\mathrm{d}}$ with moderate levels of sequence divergence between haplotypes, which suggests the accumulation of mutations in a rapidly growing population (Rogers and Harpending 1992). Unusually low nucleotide diversity exhibited within T. thynnus may have resulted from a small sample size or artificial overexploitation.

Assuming a sequence divergence of Cyt $b$ in bony fishes of $c a$. $1.0 \% \sim 1.8 \%$ per million years (Bermingham et al. 1997; Banford et al. 2004), we roughly estimated the times of species speciation to be $0.35 \sim 0.63$ Mya for T. orientalis and $0.328 \sim 0.59$ Mya 
(a)

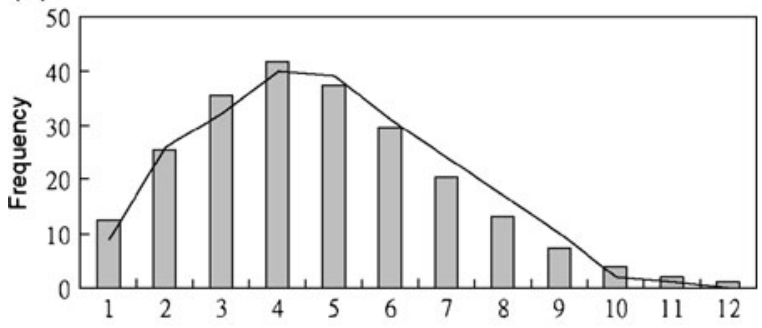

(b)

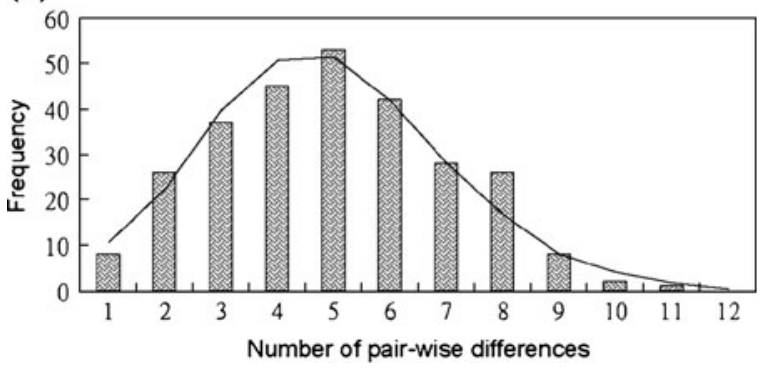

Fig. 5 Mismatch distributions obtained from mtDNA cytochrome $b$ data. The bars of the histogram represent the observed pairwise differences. The curve is the expected distribution under a sudden expansion model. a Pacific bluefin tuna. b Southern bluefin tuna

for T. maccoyii. These inferences are similar to the results of Chow and Kishino (1995) who suggested that T. maccoyii diverged about 0.5 Mya.

Thunnus orientalis and T. maccoyii have undergone historical expansion in the North Pacific and
Indian Oceans based on mismatch, Tajima's $D$, and Fu's $F_{\mathrm{s}}$ analyses, and tests of significance were used to assess the historical demographics (Fig. 5). Furthermore, the expansion times of $T$. orientalis and $T$. maccoyii were estimated to have been during the middle Pleistocene $(0.126 \sim 0.781$ Mya). There is increasing evidence that Pleistocene environmental changes, including sea level changes associated with glacial cycles (Haq et al. 1987), may have led to population expansions of marine organisms in the North Pacific and Indian Oceans. The MSTs from the Cyt $b$ gene of both species also present net-shaped trees and most haplotypes being located at the tips of the MSTs suggests adaptative radiations of the populations (Fig. 6). In addition to T. orientalis and T. maccoyii, the mismatch distribution, Tajima's $D$, and Fu's $F_{\mathrm{s}}$ analyses also provided evidence of population expansion of the Cortez bonefish Albula sp. in the Gulf of California dating to the Pleistocene (Pfeiler et al. 2008).

The nucleotide diversity between T. maccoyii and T. thynnus (reference sequences) ranged 0.0061 0.0149 which overlaps with the intraspecific nucleotide diversity of $T$. maccoyii $(0.0009 \sim 0.0123)$, suggesting a closer evolutionary relationship between these two species (Fig. 2). Nevertheless, a reverse result occurred in the analysis of amino acid sequences which showed a higher amino acid similarity between T. thynnus and T. orientalis (Fig. 4). The
Fig. 6 Minimum spanning network of a 22 haplotypes of Thunnus orientalis and $\mathbf{b}$ 22 haplotypes of T. maccoyii reconstructed by nucleotide sequences of the cytochrome $b$ gene. The size of the circles indicates the number of repetitive units belonging to the same haplotype. The length of the branches indicates the numbers of substitutions (a)
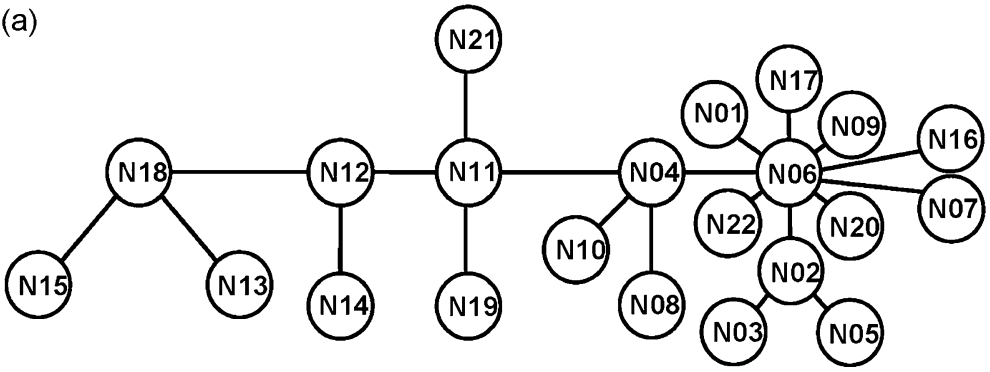

(b)

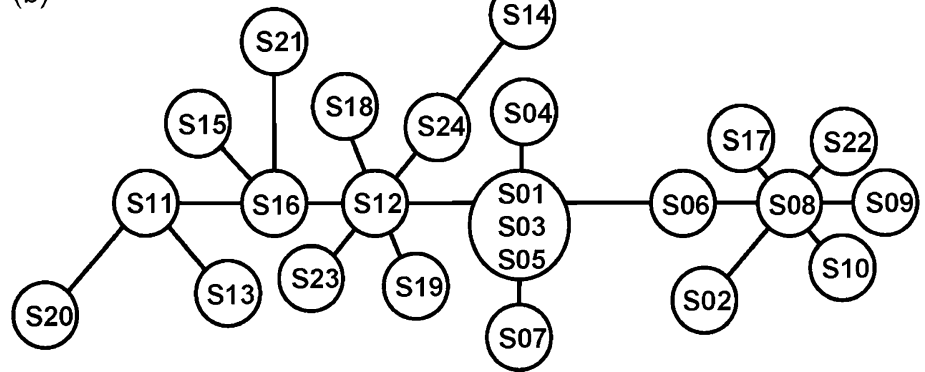


present results of amino sequences are consistent with allozyme analyses by Sharp and Pirages (1978) and Elliott and Ward (1995), indicating a closer affinity of T. orientalis to T. thynnus. However, phylogenetic relationships based on nucleotide and amino acid sequences of these three bluefin tunas remain controversial. Chow et al. (2006) examined nucleotide sequence variations of ribosomal (r)DNA ITS1 and mtDNA data from all Thunnus species, and proposed mitochondrial introgression between species. From our results, analyses of nucleotide and amino acid sequences exhibited different phylogenetic relationships among the three bluefin tunas, and reflect how the expressions of genes are affected by the environment. Silent mutations are the major variation of the Cyt $b$ gene between $T$. orientalis and $T$. thynnus, which maintains similar protein structures and functions.

Natural hybridization is believed to be more common in fish than in any other group of vertebrates (Jansson et al. 2006), and a number of gene introgressions were reported in fish (Smith 1992). Nuclear (n)DNAs of T. orientalis and T. thynnus are almost identical, but the mtDNAs greatly differ (Chow and Kishino 1995). Our results also present very dissimilar nucleotide compositions of the Cyt $b$ gene between $T$. orientalis and $T$. thynnus. A high similarity of mtDNA between $T$. orientalis and $T$. alalunga suggests that interspecific hybridization occurred between those Thunnus species (Chow and Kishino 1995). Ranges of inter- and intraspecific discrepancies of mtDNA overlapped between $T$. maccoyii and T. thynnus in this study, but a significant difference occurred in an analysis of rDNA (Chow et al. 2006). These results indicate that $T$. thynnus and T. maccoyii shared a close common female ancestor, but $T$. thynnus and $T$. orientalis shared a close common male ancestor. This implies that T. thynnus may be a hybrid species which was derived after the appearance of T. orientalis and T. maccoyii. Chow et al. (2006) explained that male-biased gene flow is quite unlikely because very few $T$. orientalis individuals have been caught in the southern hemisphere. When we explored the past demographic history, we discovered population expansion of T. orientalis (since $0.1328 \mathrm{Mya}$ ) and T. maccoyii (since $0.1868 \sim 0.2242$ Mya) during the middle Pleistocene. Distributions of marine species during interglacial and glacial periods depended on the availability of suitable current systems and the ecological characteristics of the species. However, we do not have accurate knowledge of the distribution of either species during that period. We cannot judge whether their distributions overlapped or not. From previous molecular evidence and results of this study, we inferred that historical interspecific hybridization may have taken place between these two species. More studies on tuna cytogenetics are required in order to clarify the evolutionary relationships of tuna species in the future.

Fish species identification has become an important concern, especially for high-value tuna species which are usually distributed as filleted, broiled, and canned products. Genetic markers play important roles in species identification when external morphological characteristics of fish are not available. In this study, we confirmed that the Cyt $b$ gene is very useful for discriminating the cryptic species of T. thynnus, $T$. orientalis, and T. maccoyii. We also explored the historical demography of the latter two species and discovered recent population expansions dating to the middle Pleistocene.

Acknowledgements We are extremely grateful to S.L. Lin and N.C. Chen for their help with laboratory work. This study was financially supported by a grant (96AS-15.1.2-FA-F2 (11)) from the Taiwan Fishery Agency to J.C. Shiao.

\section{References}

Akimoto S, Itoi S, Sezaki K, Borsa P, Watabe S (2006) Identification of alfonsino, Beryx mollis and B. splendens collected in Japan, based on the mitochondrial cytochrome $b$ gene, and their comparison with those collected in New Caledonia. Fish Sci 72:202-207

Alvarado-Bremer JR, Stequert B, Robertson NW, Ely B (1998) Genetic evidence for inter-oceanic subdivision of bigeye tuna (Thunnus obesus Lowe) populations. Mar Biol 132:547-557

Anonymous (1994) NBT or SBT? That is the question! Aust Fish 53:27-28

Avise JC (2000) Phylogeography: the history and formation of species. Harvard Univ. Press, Cambridge

Banford HM, Bermingham E, Collette BB (2004) Molecular phylogenetics and biogeography of transisthmian and amphi-Atlantic needlefishes (Belonidae: Strongylura and Tylosurus): perspectives on New World marine speciation. Mol Phylogenet Evol 31:833-851

Bayliff WH (1994) A review of the biology and fisheries for northern bluefin tuna, Thunnus thynnus, in the Pacific Ocean. In: Shomura RS, Majkowski J, Langi S (eds) Interactions of Pacific Tuna Fisheries. Vol. 2. Papers on Biology and Fisheries, vol 2. FAO Fisheries Technical Paper no. 336/2, Food and Agriculture Organization, Rome, pp 244-295 
Bayliff WH (2001) Status of bluefin tuna in the Pacific Ocean. IATTC, status of Pacific tuna and billfish stocks in 1999. Inter-American Tropical Tuna Commission, Stock Assessment Report 1. La Jolla, CA. pp 211-241

Bermingham E, MacCafferty S, Martin AP (1997) The isthmus of Panama, molecular clocks, and the historical biogeography of Neotropical freshwater fishes. In: Kocher TD, Stepien C (eds) Molecular systematics of fishes. Academic, New York

Bottero MT, Dalmasso A, Cappelletti M, Secchi C, Civera T (2007) Differentiation of five tuna species by a multiplex primer-extension assay. J Biotechnol 129:575-580

Cascorbi A (2003) Seafood watch seafood report: Tunas-Volume V. Monterey Bay Aquarium. (http://www.montereybayaqua rium.org/cr/cr_seafoodwatch/contentmedia/MBA_Seafood WatchSouthernBluefinTunaReport.pdf)

Caton AE (1991) Review of aspects of southern bluefin tuna biology, population and fisheries. In IATTC, World Meeting on Stock Assessment of Bluefin Tunas: Strengths and Weaknesses. Inter-Am Trop Tuna Comm Special Rep 7: pp 181-350

Chow S, Kishino H (1995) Phylogenetic relationships between tuna species of the genus Thunnus (Scombridae: Teleostei): inconsistent implications from morphology, nuclear and mitochondrial genomes. J Mol Evol 41:741-748

Chow S, Okamoto H, Miyabe N, Hiramatsu K, Barut N (2000) Genetic divergence between Atlantic and Indo-Pacific stocks of bigeye tuna (Thunnus obesus) and admixture around South Africa. Mol Ecol 9:221-227

Chow S, Nakagawa T, Suzuki N, Takeyama H, Matsunaga T (2006) Phylogenetic relationships among Thunnus species inferred from rDNA ITS1 sequence. J Fish Biol 68:24-35

Collette BB (1999) Mackerels, molecules, and morphology. In: Seret B, Sire J-Y (eds) Proceedings of the 5th Indo-Pacific Fish Conference, Noumea 1997. Société Française d'Ichtyologie, Paris, pp 149-164

Collette BB, Nauen CE (1983) FAO species catalogue. Vol. 2. Scombrids of the world. An annotated and illustrated catalogue of tunas, mackerels, bonitos and related species known to date. FAO Fish Synop 125:1-37

Collette BB, Smith BR (1981) Bluefin tuna, Thunnus thynnus orientalis, from the Gulf of Papua. Jpn J Ichthyol 28:166168

Collette BB, Reeb C, Black BA (2001) Systematics of the tunas and mackerels (Scombridae). In: Block BA, Stevens ED (eds) Tuna: physiology, ecology, and evolution. Academic, San Diego, pp 1-33

Elliott NG, Ward RD (1995) Genetic relationships of eight species of Pacific tunas (Teleostei: Scombridae) inferred from allozyme analysis. Mar Freshw Res 46:1021-1032

Excoffier L, Laval LG, Schneider S (2005) Arlequin vers. 3.0: an integrated software package for population genetics data analysis. Evol Bioinform Online 1:47-50

Fu YX (1997) Statistical tests of neutrality of mutations against population growth, hitchhiking and background selection. Genetics 147:915-925

Gibbs RH Jr, Collette BB (1967) Comparative anatomy and systematics of the tunas, genus Thunnus. US Fish Wildl Serv Fish Bull 66:65-130

Gunn JS, Clear NP, Carter TI, Rees AJ, Stanley CA, Farley JH, Kalish JM (2008) Age and growth in southern bluefin tuna, Thunnus maccoyii (Castelnau): direct estimation from otoliths, scales and vertebrae. Fish Res 92:207-220

Haq BU, Hardenbol J, Vail PR (1987) Chronology of fluctuating sea levels since the Triassic. Science 235:1156-1167

Harpending H (1994) Signature of ancient population growth in a low-resolution mitochondrial DNA mismatch distribution. Hum Biol 66:591-600

Hattour A, Macías D (2002) Bluefin tuna maturity in Tunisian waters: a preliminary approach. Col Vol Sci Pap ICCAT 54:545-553

Hewitt GM (2000) The genetic legacy of the Quaternary ice ages. Nature 405:907-913

Hudson RR (1990) Genealogies and the coalescent process. In: Furuyama, Antonovics JD (eds) Oxford surveys in evolutionary biology. Oxford University Press, New York, pp $1-44$

Jansson H, Holmgren I, Wedin K, Anderson T (2006) High frequency of natural hybrids between Atlantic salmon, Salmo salar L., and brown trout, S. trutta L., in a Swedish river. J Fish Biol 39:343-348

Kocher TD, Thomas WK, Meyer A, Edwards SV, Pabo S, Villablabca FX, Wilson AC (1989) Dynamics of mitochondrial DNA evolution in animals: amplification and sequencing with conserved primers. Proc Natl Acad Sci USA 86:6196-6200

Li WH (1997) Molecular evolution. Sinauer, Sunderland

Manchado M, Catanese G, Infante C (2004) Complete mitochondrial DNA sequence of the Atlantic bluefin tuna Thunnus thynnus. Fish Sci 70:68-73

Mather FJ, Mason JM, Jones AC (1995) Historical document: life history and fisheries of Atlantic bluefin tuna. NOAA Technical Memorandum 370 NOAA, Miami, FL.

Nei M, Kumar S (2000) Molecular evolution and phylogenetics. Oxford University Press, New York. NOAA http:// www.noaa.gov/fisheries.html

Pfeiler E, Watts T, Pugh J, van der Heiden AM (2008) Speciation and demographic history of the Cortez bonefish, Albula sp. A (Albuliformes: Albulidae), in the Gulf of California inferred from mitochondrial DNA. J Fish Biol 73:382-394

Planes S, Doherty PJ, Bernardi G (2001) String genetic divergence among populations of a marine fish with limited dispersal, Acanthochromis polyacanthus, within the great barrier reef and the coral sea. Evolution 55:22632273

Porch CE (2005) The sustainability of western Atlantic bluefin tuna: a warm-blooded fish in a hot-blooded industry. Bull Mar Sci 76:363-384

Posada D, Crandall KA (1998) MODELTEST: testing the model of DNA substitution. Bioinformatics 14:817-818

Rogers AR (1995) Genetic evidence for a Pleistocene population explosion. Evolution 49:608-615

Rogers AR, Harpending H (1992) Population growth makes waxes in the distribution of pairwise genetic differences. Mol Biol Evol 9:552-569

Rohlf FJ (1973) Algorithm 76. Hierarchical clustering using the minimum spanning tree. Comput J 16:93-95

Schneider S, Excoffier L (1999) Estimation of past demographic parameters from the distribution of pairwise differences when the mutation rates vary among sites: application to human mitochondrial DNA. Genetics 152:1079-1089 
Sharp GD, Pirages S (1978) The distribution of red and white swimming muscles, their biochemistry, and the biochemical phylogeny of selected scombrid fishes. In: Sharp GD, Dizon AE (eds) The physiological ecology of tunas. Academic, New York, pp 41-78

Shiao JC, Chang SK, Lin YT, Tzeng WN (2008) Size and age composition of southern bluefin tuna (Thunnus maccoyii) in the central Indian Ocean inferred from fisheries and otolith data. Zool Stud 47:158-171

Shiao JC, Yui TF, Høie H, Ninnemann U, Chang SK (2009) Otolith $\mathrm{O}$ and $\mathrm{C}$ stable isotope composition of southern bluefin tuna Thunnus maccoyii (Pisces: Scombridae) as possible environmental and physiological indicators. Zool Stud 48:71-82

Slatkin M, Hudson R (1991) Pairwise comparisons of mitochondrial DNA sequences in stable and exponentially growing populations. Genetics 129:555-562

Smith GR (1992) Introgression in fishes: significance for paleontology, cladistics, and evolutionary rates. Syst Biol 41:41-57

Smith PJ, Conroy AM, Taylor PR (1994) Biochemical-genetic identification of northern bluefin tuna Thunnus thynnus in the New Zealand fishery. NZ J Mar Freshw Res 28:113118

Smith PJ, Griggs L, Chow S (2001) DNA identification of Pacific bluefin tuna (Thunnus orientalis) in the New Zealand fishery. NZ J Mar Freshw Res 35:843-850

Tajima F (1989) Statistical method for testing the neutral mutation hypothesis by DNA polymorphism. Genetics 123:585-595

Tajima F (1996) The amount of DNA polymorphism maintained in a finite population when the neural mutation rate varies among sites. Genetics 143:1457-1465

Takehana Y, Uchiyama S, Matsuda M, Joen SR, Sakaizumi M (2004) Geographic variation and diversity of the cytochrome $b$ gene in wild populations of medaka
(Oryzias latipes) from Korea and China. Zool Sci 21:483-491

Takeyama H, Chow S, Tsuduki H, Matsunaga T (2001) Mitochondrial DNA sequence variation within and between Thunnus tuna species and its application to species identification. J Fish Biol 58:1646-1657

Tamura K, Dudley J, Nei M, Kumar S (2007) MEGA4: molecular evolutionary genetics analysis (MEGA) software vers. 4.0. Mol Biol Evol 24:1596-1599

Thompson JD, Higgins DG, Gibson TJ (1994) CLUSTAL W: Improving the sensitivity of progressive multiple sequence alignment through sequence weighting, position specific gap penalties and weight matrix choice. Nucleic Acids Res 22:4673-4680

Tseng MC, Jean CT, Tsai WL, Chen NC (2009) Distinguishing between two sympatric Acanthopagrus species from Dapeng Bay, Taiwan, using morphometric and genetic characters. J Fish Biol 74:357-376

Ward RD, Elliott NG, Grewe PM (1995) Allozyme and mitochondrial DNA separation of Pacific northern bluefin tuna, Thunnus thynnus orientalis (Temminck and Schlegel), from Southern bluefin tuna, Thunnus maccoyii (Castelnau). Mar Freshw Res 46:921-930

Ward RD, Elliott NG, Innes BH, Smolenski AJ, Grewe PM (1997) Global population structure of yellowfin tuna, Thunnus albacares, inferred from allozyme and mitochondrial DNA variation. Fish Bull 95:566-575

Wu GCC, Chiang HC, Chen KS, Hsu CC, Yang HY (2009) Population structure of albacore (Thunnus alalunga) in the northwestern Pacific Ocean inferred from mitochondrial DNA. Fish Res 95:125-131

Zhao L, Zhang J, Liu Z, Funk SM, Wei F, Xu M, Li M (2008) Complex population genetic and demographic history of the salangid, Neosalanx taihuensis, based on cytochrome b sequences. BMC Evol Biol 8:201. doi:10.1186/1471-21488-201 